International Journal of Pure and Applied Mathematics

Volume 82 No. 4 2013, 663-668

ISSN: 1311-8080 (printed version); ISSN: 1314-3395 (on-line version)

url: http://www.ijpam.eu

doi: http://dx.doi.org/10.12732/ijpam.v82i4.14

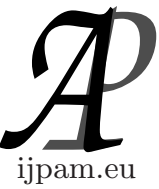

\title{
MODELING OF DENDRITIC GROWTH IN TWO DIMENSIONS
}

\author{
G.S. Dmitrienko ${ }^{1 \S}$, L.A. Uvarova ${ }^{2}$ \\ Department of Applied Mathematics \\ MSUT STANKIN \\ 3a, Vadkovsky Lane., Moscow, 127055, RUSSIA
}

\begin{abstract}
The paper describes the process of growth of two-dimensional structures on the basis of the phase-field model and the DLA (Diffusion-limited aggregation). We consider a hybrid model, based on which can be described various transport phenomena in systems of dispersed particles, nanoparticles, etc. Investigated the characteristic parameters of the model. Give estimates of the rate of growth of structures described by such models.
\end{abstract}

Key Words: phase-field model, diffusion-limited aggregation

\section{Introduction}

Mathematical modeling of heat and mass transfer in systems of dispersed particles, nanoparticles and nanofluids with magnetic properties is one of actual problems at present. This paper considers the hybrid model based on the phase-field model and the DLA, which can be used to describe such processes.

There are many modifications of the algorithm DLA [1-3]. Structure resulting from the operation of such an algorithm similar to crystals, solidified

Received: November 27, 2012

(c) 2013 Academic Publications, Ltd. url: www.acadpubl.eu

${ }^{\S}$ Correspondence author 
melt or salts, and other objects found in nature [2-3]. Analogous algorithm can be used to model the dielectric breakdown and other processes in systems with magnetic properties [3]. Described on this approach, the structure have fractal properties $[2-4]$.

In [7-12] describes the phase-field model, widespread at present. Form of the surface structures in these models is changed due to the phase transformation, taking into account the anisotropy of the surface tension. In [9-11] are considered and studied the modification of such models with the deposition of particles on the surface.

\section{Modelling}

Building a cluster DLA is based on numerical methods. Original algorithm consists of the following steps:

1. On a square two-dimensional grid at the origin is the initial particle.

2. Away from the cluster is born a new particle.

3. New particle wanders randomly (for simplicity, we assume that the particle can move in 4 directions).

4. If the particle is conveniently situated close to completed cell, then it sticks.

5. If the particle moves away from the cluster, it disappears.

6. Repeat - from step 3 as long as the particle does not stick or did not die, then there is a start of a new particle (step 2).

The output of the algorithm depends on the step 2 - that is, that where there is a new particle. Often, the implementation of such an algorithm is given a circle with a radius that is much superior to the particle size and the center of the initial particle, as well as changing the radius of the circle. At such a circle randomly appear particles. In Figure 1. shows a typical DLA-clusters.

The advantage of the model described in this paper is the consideration of mechanisms of particle deposition and growth of structure through the phase transition. This model can be applied to a wider class of systems that need to take into account both the growth mechanism. In another paper considers a similar model for the formation of ice crystals on surfaces (patterns), various approaches to the modeling of these processes, including DMB and DLA. The algorithm described in this paper is a generalization.

The crystallization process with moving interphase boundaries will be considered on the basis of models that have been studied in [7-11]. These models 


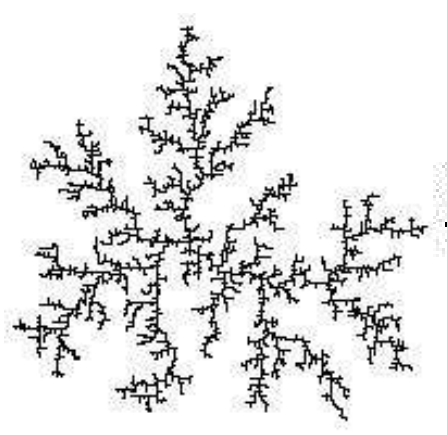

a)

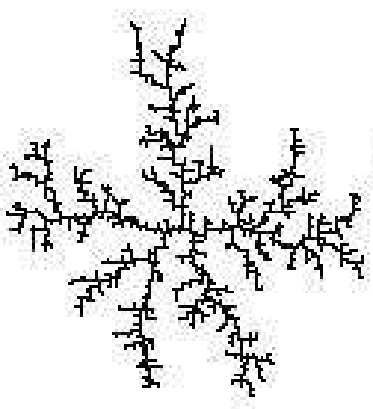

b)

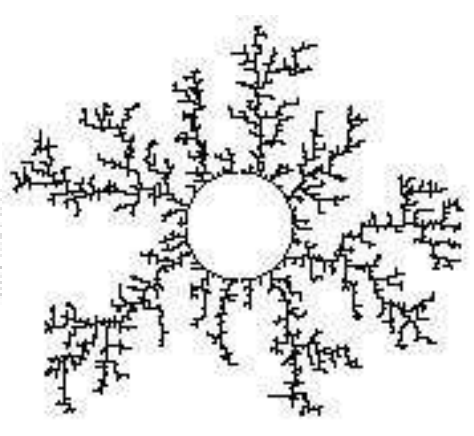

c)

Figure 1: The structures were generated by the algorithm DLA: (a) the particles appear on a circle of constant radius, (b) - the particles appear in a circle of radius changing, (c) - the particles are deposited on the circle, and appear on a circle of constant radius.

are based on the introduction of the spatial variable phase field $p(x, y, t)$ as a function of horizontal and vertical coordinates $x$ and $y$ with time $t$, which characterizes the state of matter, and taking different values for the solid and liquid phases (1 and 0$)$.

$$
\tau \frac{\partial p}{\partial t}=\nabla\left(\varepsilon^{2} \nabla p\right)+p(1-p)\left(p-\frac{1}{2}+m\right)
$$

For the dimensionless temperature $T(x, y, t)$ equation can be written

$$
\frac{\partial T}{\partial t}=\nabla^{2} T+K \frac{\partial p}{\partial t}
$$

Value of the variables and constants are taken in a similar way as described in $[7-11]$.

Consider a hybrid model. The hybrid model includes mechanisms of the models described above. In the phase-field model with the given parameters $p(x, y, t)$ and $T(x, y, t)$ and initial conditions, and in the form of a circular particle [11]. On a circle of a given radius centered at the round particles nucleate small particles. Small particles wander in the steps of the algorithm described above. Sticking to the growing structure is the case, if the particle is near the square, where the value $p(x, y, t)$ is greater than the critical value $p_{0}$. The velocity of the particle is determined by the size $\delta_{0}$ (number of random walks in a unit of time). 
Deposition of particles on the surface alters the growth rate of the entire structure because the rate of growth of structure in the phase-field model greatly depends on the curvature of the surface. In Fig. 2 shows the structure of growth.

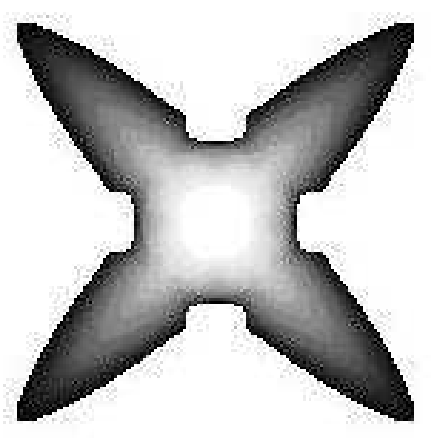

a)

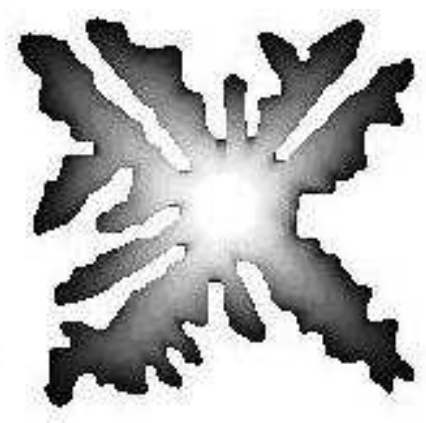

b)

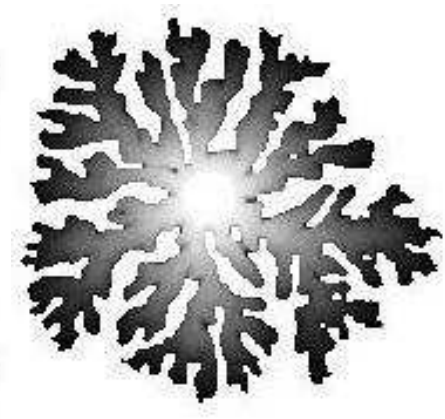

c)

Figure 2: Growth structure were generated: (a) - the phase-field model, (b) - a hybrid model for $\delta_{0}=0.6, p_{0}=0.5$, and (c) - a hybrid model for $\delta_{0}=6, p_{0}=0.5$. Different colors correspond to different ages, white color - initial time, black color - the final time.

To study the value of the model is investigated numerically in the size of the structure over time. Such a value may be defined by the form:

$$
H(t)=\int_{0}^{H_{\mathrm{x}}} \int_{0}^{H_{\mathrm{y}}} p(x, y, t) d x d y
$$

In Fig. 3 shows the typical dependence $H(t)$.

For values of the parameter $\mathrm{K}$ is about 1.5 (phase-field model parameter), the dependence $H(t)$ of the structure of phase-field model for the initial time interval can be considered linear, as shown in [11]. In the hybrid model, the growth rate will depend on the probability of deposition of particles on the surface (the more particles join - the more structure), due to the fact that the particle can not adhere to the structure. Probability of settling particles on the surface depends on the size and shape of the structure. Therefore, as a first approximation, we can assume that the growth rate is directly proportional to the size of the structure. Which implies that over time the size of the structure increases exponentially. What is clear from the graphs. 


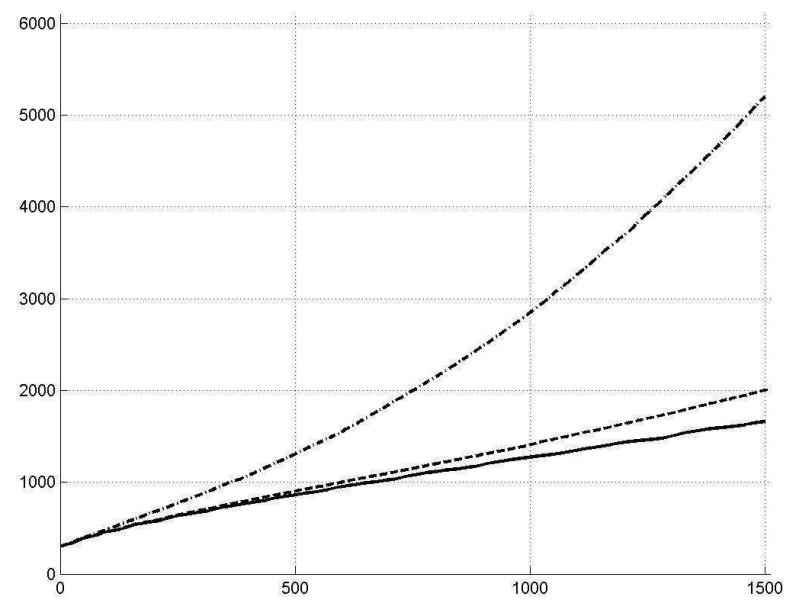

Figure 3: The figure shows the dependence $H(t)$ : solid line for the phase-field model, dotted line for the hybrid model $\delta_{0}=3 \cdot 10^{3}$, dotted line with a point for the hybrid model $\delta_{0}=3 \cdot 10^{4}$.

\section{Conclusion}

In work was considered a hybrid model that can be used for modeling of processes with a moving boundary, such as the crystallization of the deposition of particles on the surface, in the systems of dispersed particles, nanoparticles and nanofluids. Simulation results are estimates of the value of the growth rate.

\section{References}

[1] T.A. Witten, L.M. Sander, Diffusion-limited aggregation, a kinetic critical phenomenon, Phys. Rev. Letters., 47 (1981), 1400.

[2] A.Yu. Menshutin, L.N. Shchur, V.M. Vinokour, Finite size effect of harmonic measure estimation in a DLA model: Variable size of probe particles, Physica A, 387, No. 25 (2008), 6299-6309.

[3] L. Niemeyer, L. Pietronero, H.J. Wiesmann, Fractal dimension of dielectric breakdown, Phys. Rev. Lett., 52 (1984), 1033-1036.

[4] B. Mandelbrot, The Fractal Geometry of Nature, WH Freeman and Co., ISBN 0716711869 (1982). 
[5] A.-L. Barabasi, H.E. Stanley, Fractal Concepts in Surface Growth, Cambridge Univesity Press (1995).

[6] F. Family, T. Vicsek, Dynamics of Fractal Surfaces, World Scientific Publishing Co.Pte.Ltd., ISBN 981-02-0720-4 (1991).

[7] R. Kobayashi, Modeling and numerical simulations of dendritic crystal growth, Physica D: Nonlinear Phenomena, 63, No-s: 3-4 (1993), 410-423.

[8] T. Won-Hyung Kim, Physically-Based Simulation of Ice Formation, Dissertation, University of North Carolina, Chapel Hill (2006).

[9] G. Dmitrienko, L. Uvarova, Modeling of heat transfer in an area with a fractal boundary, Vestnik MSTU "Stankin", 2, No. 2 (2012), 50-54, In Russian.

[10] G.S. Dmitrienko, L.A. Uvarova, Phase field modeling of moving surface roughness profile, International Journal of Pure and Applied Mathematics, 75, No. 1 (2012), 93-102.

[11] G.S. Dmitrienko, Modeling of Heat Transfer Processes in Systems with Moving Rough Boundaries on the Basis of the Theory of Phase Field, Moscow, Yanus-K (2012), In Rusian.

[12] M. Benes, Computation studies of anisotropic diffuse interface model of microstructure formation in solidification, Acta Math. Univ. Comenianae, Proceedings of Equadiff 11, LXXVI, No. 1 (2007), 39-50. 